\title{
Binding of Anaphors in LTAG
}

\author{
Neville Ryant \\ Department of Linguistics \\ 619 Williams Hall \\ University of Pennsylvania \\ Philadelphia, PA, 19104-6305 \\ nryanteling . upenn . edu
}

\author{
Tatjana Scheffler \\ Department of Linguistics \\ 619 Williams Hall \\ University of Pennsylvania \\ Philadelphia, PA, 19104-6305 \\ tat jana@ling. upenn. edu
}

\begin{abstract}
This paper presents an LTAG account for binding of reflexives and reciprocals in English. For these anaphors, a multi-component lexical entry is proposed, whose first component is a degenerate NP-tree that adjoins into the anaphor's binder. This establishes the local structural relationship needed to ensure coreference and agreement. The analysis also allows a parallel treatment of reflexives and reciprocals, which is desirable because their behavior is very similar.

In order to account for non-local binding phenomena, as in raising and ECM cases, we employ flexible composition, constrained by a subject intervention constraint between the two components of the anaphor's lexical entry. Finally, the paper discusses further data such as extraction and picture-NP examples.
\end{abstract}

\section{Introduction}

Binding Theory (Büring, 2005; Reuland and Everaert, 2001) is an issue at the interface of syntax and semantics which has previously been avoided in the LTAG literature. While LTAGs were initially concerned only with the syntax of natural languages, recent accounts of semantic computation in the LTAG framework (Kallmeyer and Joshi, 2003; Kallmeyer and Romero, 2004) allow us now to tackle interface phenomena. An appropriate formulation of Binding Theory (BT) is needed to explain the pattern exhibited in (1-3).

(1) $\operatorname{John}_{i}$ likes himself $_{i}$.

(2) $* \mathrm{John}_{i}$ likes herself $_{i}$.
(3) $*$ Himself $_{i}$ likes himself $i / \operatorname{John}_{i}$.

Due to the incredible complexity of the data in question, we will focus here on English reflexives (himself, herself) and reciprocals (each other), typically subsumed under Condition A (Chomsky, 1981).

This paper proposes a new two-component lexical entry for reflexive pronouns that takes care of the syntactic and semantic dependencies involved in binding (agreement and coreference). In this approach, different binding options (e.g., in a ditransitive sentence) follow from different derivations.

In section 3, we show how our analysis extends straightforwardly to reciprocals. Section 4 presents the extension of our account to anaphors with nonlocal antecedents, such as the experiencers of raising verbs, and ECM subjects. Further issues, including extraction, are discussed in section 5. Section 6 concludes.

\section{Basic Anaphor Binding}

In traditional accounts, binding is defined representationally: an antecedent binds an anaphor iff they are are coindexed and in a certain structural relationship. In an LTAG, binding cannot be viewed in this way as the notion of coindexation is foreign to the formalism. An LTAG analysis can therefore not be a mere translation of a previous account.

Although the phenomenon is very complex, the basic properties of binding are quite well understood. Binding of an anaphor by an antecedent consists of coreference and agreement between the two items. Furthermore, it is well known that binding of English anaphors is an asymmetrical, local, structural relationship. The asymmetry of binding can be easily observed in examples (1) 
versus (3). Locality is reflected by the fact that (1) is grammatical, but not (4).

(4) $* \mathrm{John}_{i}$ knows that Mary likes himself ${ }_{i}$.

Finally, the binding relationship is known to be structural because the positions of binder and anaphor play a crucial role. This is discussed in more detail below.

\subsection{Lexical Entry}

The domain of locality that LTAG provides enables us to encode a local structural relationship, such as the one between the anaphor and its antecedent, very directly. We understand binding as a lexical requirement of the anaphor: that it must be bound. Thus, we propose the lexical entry in Figure 1 for reflexives. It is a multicomponent set whose second component is the anaphor. The first component is a degenerate auxiliary tree which adjoins into the elementary tree of the antecedent.

In LTAG, elementary trees encode both syntax and semantics. Thus, the two components of binding, coreference and agreement, are simultaneously guaranteed by the coindexations between the feature structures of binder and anaphor. Furthermore, since the derivation must be tree-local, locality is also ensured. A c-command constraint between the two components accounts for the asymmetry between the antecedent and anaphor as shown in examples (1) and (3). This constraint is checked when the two components are composed into an elementary tree (by tree-locality).

\subsection{Example Derivation}

Consider (5), where himself has two possible antecedents, John and Bill. Our analysis derives both readings, given a standard tree inventory as in Figure 2.

(5) $\operatorname{John}_{i}$ showed Bill $_{j} \operatorname{himself}_{i / j}$.

Sentence (5) is syntactically ambiguous under this analysis, since two different derivations lead to distinct readings. This seems to reflect our intuitions about this sentence well, although it contrasts with the traditional vew of BT, where the coindexation between binder and anaphor is part of the syntactic structure for the sentence, and thus no ambiguity arises.

\subsection{Flexible Composition}

Tree-locality requires the first component of himself to adjoin into a higher NP substitution node.

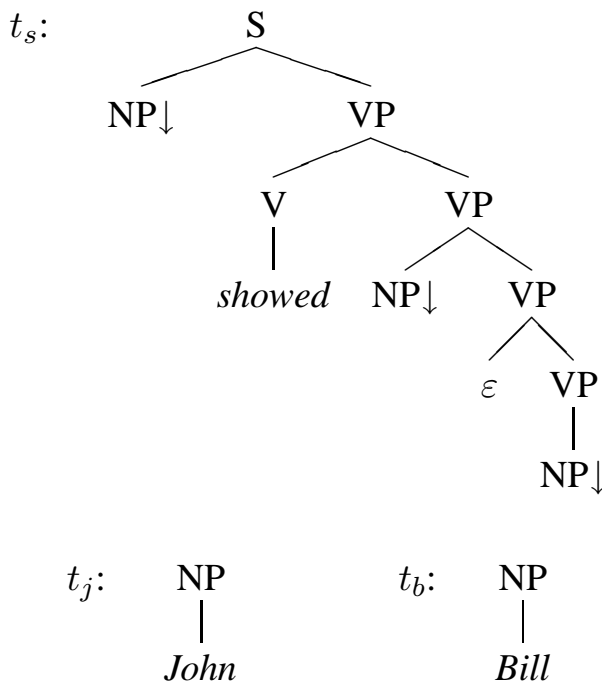

Figure 2: Tree inventory.

However, adjunction into substitution nodes is generally disallowed. Adjunction of the first component of himself into the root node of the Johntree $t_{j}$ or the Bill-tree $t_{b}$ is, however, not tree-local. Therefore, we employ flexible composition (Joshi et al., 2003) to compose $t_{j}$ with the first component of $t_{h}\left(t_{h}^{1}\right)$, yielding a derived multicomponent set. Composition of $t_{h}$ with $t_{s}$ is then treelocal. This yields the reading where John is the antecedent of himself.

Alternatively, $t_{b}$ composes with $t_{h}$ first, which derives the other reading. The two derivation trees representing these readings are shown in Figure 3.

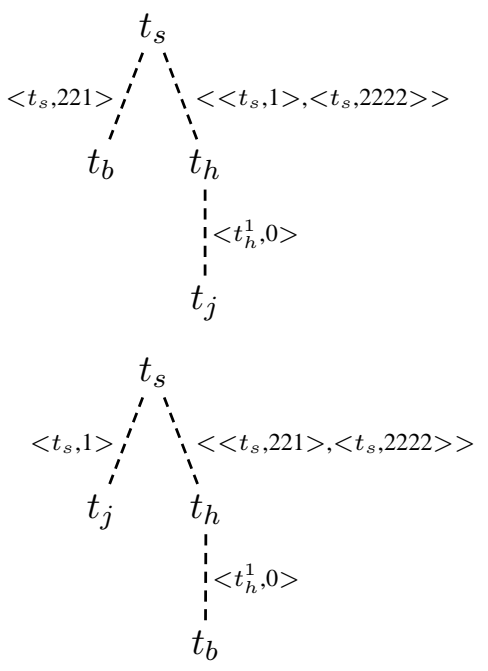

Figure 3: Derivation trees for "John showed Bill himself." 


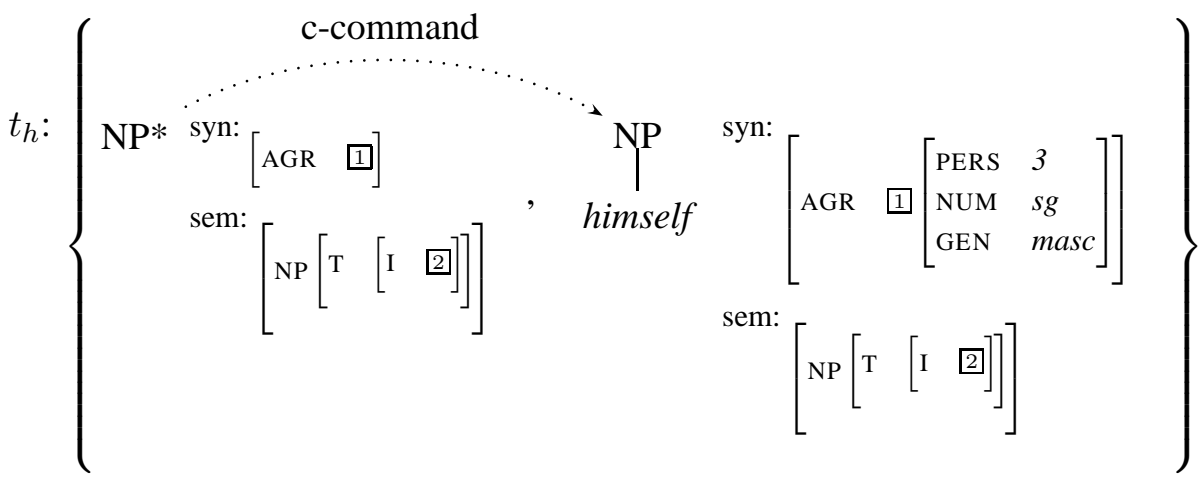

Figure 1: Lexical entry for himself.

\subsection{Advantages}

The different binding options (e.g., in doubleobject sentences) follow directly from the derivation and do not have to be hardcoded. Furthermore, the reflexive itself is responsible for agreement and coreference with its antecedent.

\subsection{Alternative Analysis}

There is at least one obvious alternative analysis for BT in LTAG. In this case, features are employed instead of a multicomponent set to derive the binding relationship. Features on each verbal elementary tree would encode whether an argument is an anaphor, and if so, what it is bound to. Just like in our analysis introduced above, a certain locality necessary for binding can be ensured under this approach. However, this approach is very stipulative. It is merely an accident that agreement and coreference go hand in hand: Two separate feature equations have to ensure agreement between the binder and anaphor, and coreference between them. Furthermore, a number of verbal trees is added; and the reflexive itself becomes syntactically and semanticially vacuous.

\section{Reciprocals}

Another advantage of the proposed account is that it allows an analogous treatment of reciprocals like each other in (6).

(6) $[\text { John and Mary }]_{i}$ like each other ${ }_{i}$.

This is desirable given that the syntactic behavior of reciprocals resembles reflexives. Semantically, though, reciprocals are very complex (Dimitriadis, 2000). The meaning of "each other" roughly corresponds to its parts, "each", and "other". That is, "John and Mary love each other" means something like "Of John and Mary, each loves the other". 1

These properties are neatly accounted for with our analysis of each other that is syntactically analogous to himself, but contributes additional operators in the semantics ${ }^{2}$. The proposed lexical entry is spelled out in Figure 4.

The fact that each other contributes two distinct quantifiers corresponds directly to its syntactic analysis as a two-part multicomponent set.

\section{Nonlocal Antecedents}

The discussion of anaphoric pronoun binding discussed in the previous section demonstrated how certain locality (7) and configurational restrictions (8) on anaphoric pronouns follow from TAG's constrained mechanisms of structural composition coupled with a multicomponent analysis of reflex-

\footnotetext{
${ }^{1}$ It is sometimes claimed that "long-distance" reciprocals require non-local adjunction of "each":

(i) The boxers thought they would defeat each other. $\checkmark$ each \# each

The LTAG analysis proposed here does not allow this. This may constitute independent evidence for Dimitriadis' (2000) analysis of reciprocals in which "each" is not as high as it seems in these kinds of examples.

${ }^{2}$ The exact semantics of each other is a matter of ongoing discussion. We assume for simplicity that each other corresponds to each+the other, as reflected in the lexical entry.

${ }^{3} \sqsubseteq A=$ "is an atomic part of".

In the absence of a complete analysis of plural semantics in LTAG, we assume here that plural noun phrases like "John and Mary" or "the boys" contribute at least a group (G) variable. This variable is used by certain collective predicates, for example in "The boys surrounded the castle." It corresponds to the plural individual contributed by the NP.

The semantics given here predicts strongly distributive "each other". Some adjustment is needed to account for lesser forms of distributivity.
} 


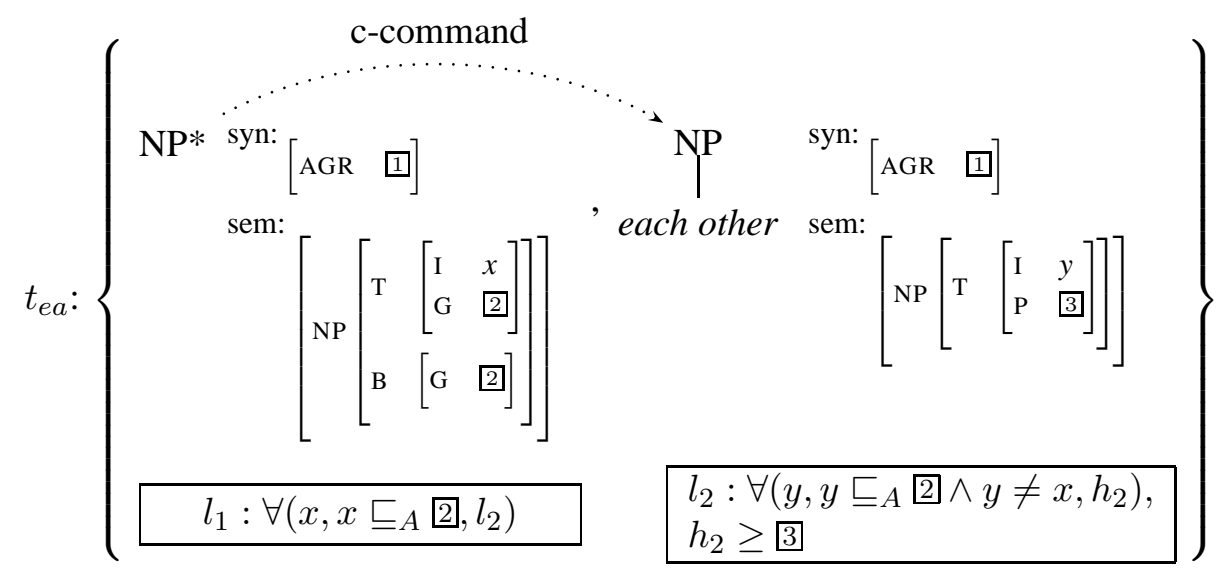

Figure 4: Lexical entry for each other. ${ }^{3}$

ives and reciprocals.

a. John $_{i}$ likes himself . $_{\text {. }}$

b. ${ }^{*} \operatorname{John}_{i}$ thinks that Mary believes that Kate likes himself ${ }_{i}$.

(8) a. John believes Mary ${ }_{i}$ to like herself ${ }_{i}$.

b. *John believes herself $i$ to like Mary . $^{*}$

A significant problem with this analysis as stands, however, is that it works too well, denying the grammaticality of certain raising (9) and ECM constructions (10) and constructions in which the anaphor is embedded within a subject (11). Under current assumptions, the antecedent-anaphor dependency must be established within an elementary tree (by adjunction of a single multicomponent set). However, for both of these constructions the anaphor and its antecedent lie in different elementary trees. In (9) the auxiliary tree of the raising verb seems contains no local argument for the degenerate NP* component to combine with. In (10) himself occurs as an argument of like while its antecedent occurs in another elementary tree, believe. In each case, generating the examples requires that we relax some of our current assumptions.

(9) $\mathrm{John}_{i}$ seems to himself $i$ to be a decent guy.

(10) $\mathrm{John}_{i}$ believes himself $i$ to be a decent guy.

(11) $\mathrm{John}_{i}$ thought that the pictures of himself $f_{i}$ were wonderful.

\subsection{Raising}

We see from (9) that anaphors can occur as experiencer arguments of raising verbs providing they are c-commanded by a possible antecedent. Though predicted to be ungrammatical under the current proposal, (9) can be generated if we relax the requirement that the two parts of the multicomponent set of the anaphor attach to the same elementary tree. This relaxation could take the form of simply allowing non-local adjunction for specific classes of multicomponent sets, those with a degenerate components. Alternately, we retain the restriction to tree-local MC-TAG but achieve nonlocality through more extensive use of flexible composition, already adopted for independent reasons.

Under a flexible composition analysis (Figure 6), the John-tree composes with the degenerate $\mathrm{NP}^{*}$ member of the reflexive set as before. This yields a derived multicomponent set consisting of one derived part, John, and one underived part, himself. The seems-tree then composes with the himself component of the reflexive set, yielding a derived set (Figure 5) containing the components John and seems to himself. Finally, this derived multicomponent set combines with the like-tree, the John component substituting into the open NP slot and the seems to himself component adjoining at VP.

\subsection{ECM}

In ECM constructions such as (10) the anaphor appears as the subject of the embedded to be a decent guy-tree while its antecedent appears as subject of the matrix believes-tree. A derivation for this sentence under our account is shown in Figure 7. As before, the John-tree first composes with the degenerate NP* component of the reflexive tree, followed by the the substitution of the himself-tree 


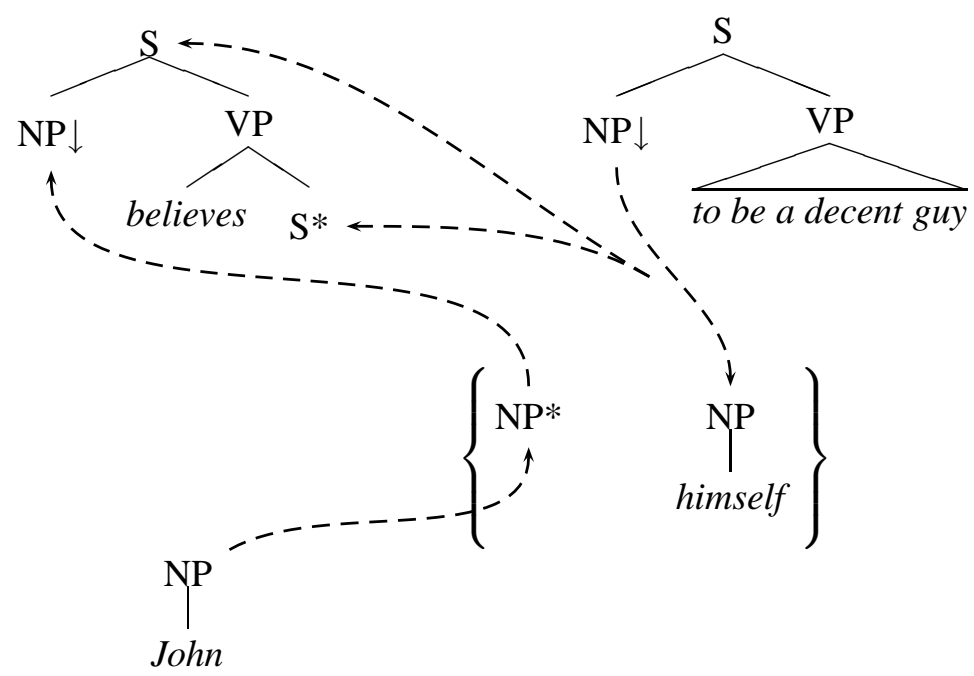

Derivation tree:

Figure 7: Derivation of "John believes himself to be a decent guy."

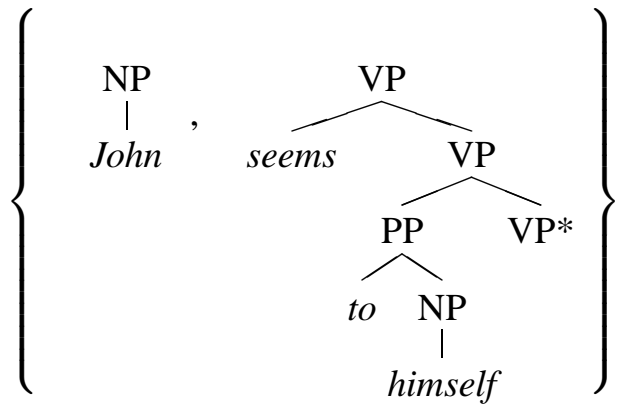

Figure 5: Derived multicomponent set for (9).

into the to be a decent guy-tree, yielding the derived multicomponent set containing John and believes himself, which locally composes with the to be a decent guy-tree.

\subsection{Subject Embedding}

Anaphors contained within embedded subjects ${ }^{4}$ (12) cause the binding domain to be minimally expanded. Again, it is transparent that these cases can be derived successfully from the lexical entry in Figure 1 and repeated use of flexible composition.
(12) a. The $\operatorname{men}_{i}$ knew that pictures of each other $i$ were on sale.
b. The $\operatorname{men}_{i}$ felt that the pictures of themselves $i$ were horrid.
c. The $\operatorname{men}_{i}$ knew that each other ${ }_{i}$ 's pictures were on sale.

\footnotetext{
${ }^{4}$ The absence of nonlocal binding of reflexive subjects (e.g. John thinks that himself is grand.) is assumed to derive from an inability of reflexives to take nominative case.
}
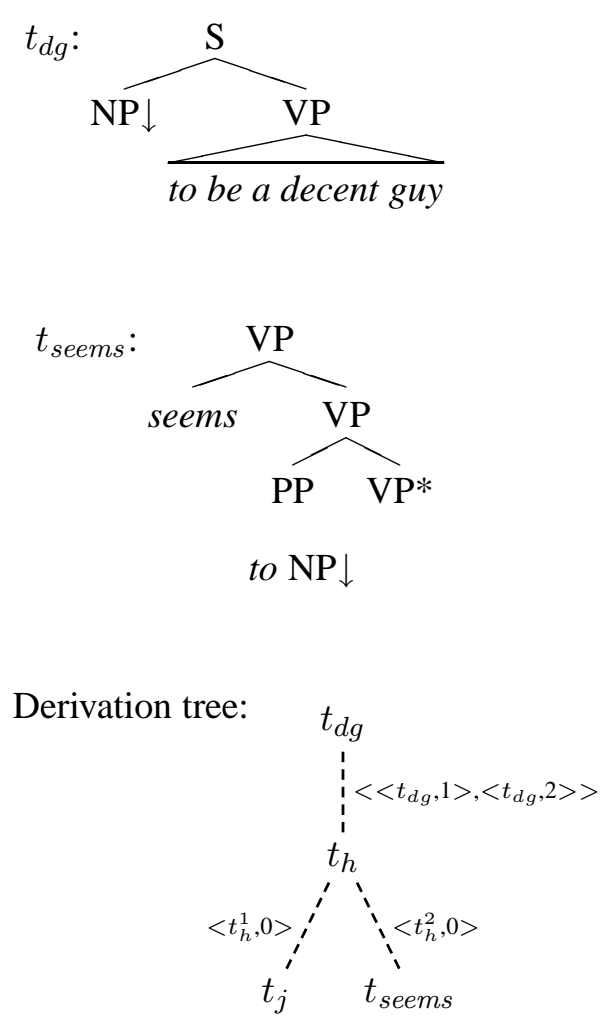

Figure 6: Derivation of "John seems to himself to be a decent guy." 


\subsection{Constraints on Flexible Composition}

The use of flexible composition with tree-local MC-TAG is very powerful, thus able to account for the non-local binding in (9), (10), and (12). However, it is too powerful if unconstrained as it will also generate (13). It is necessary to constrain the derivation such that in the derived tree no subject node intervenes between the antecedent and anaphor (Chomsky's Subject Intervention Constraint). This is obtained by strengthening the link between NP and himself in the lexical entry s.t. when the two trees connected by the link are adjoined, a requirement that $\mathrm{NP}^{*} \mathrm{c}$-command himself and no subject NP intervenes between the two (c-commanding himself and c-commanded by NP*) is checked. This constraint formalizes the descriptive account given in the linguistic literature. Note that a similar account may be active in other places in the grammar as well, due to the pervasiveness of left-edge phenomena (see section 5.4).

Computationally, this constraint can be checked as soon as the multicomponent set which contains it attaches into another elementary tree. Ccommand as well as subject intervention cannot be disturbed by later operations on the outer tree, if they are valid at the time of composition.

(13) $* \operatorname{John}_{i}$ believes me to like himself ${ }_{i}$.

\section{Further Issues}

\subsection{Exempt Pronouns}

As it currently stands, the proposal follows heavily in the footsteps of traditional configurational approaches to BT. As such, it mirrors the more traditional BT of Chomsky in it's inability to license such examples as (17b), where the antecedent does not c-command the anaphor and (14) and (15), where binding is possible despite presence of an intervening subject along the c-command path.

(14) a. I spoke to $[\text { John and Bill }]_{i}$ about each other $_{i}$.

b. Pictures of myself frighten $_{i}$. $_{\text {. }}$

c. $\mathrm{John}_{i}$ 's greatest problem is a fear of himself $i$.

(15) $[\text { John and Mary }]_{i}$ are letting the honey drip on each other ${ }_{i}$ 's feet.

(16) Clones of each other annoy the children.
The examples in (14) can be accommodated by having the prepositions appearing before the arguments be surface case markers rather than real prepositions (as suggested in (Jackendoff, 1972)). Even so, (15) and (16) remain and seem to present an intractable problem for an LTAG account, as well as traditional accounts of English binding phenomena. This may in fact be the case and prove firm support for claims by numerous authors (Pollard and Sag, 1994; Postal, 1971; Kuroda, 1965) that at least part of the data subsumed under BT (the "exempt pronouns") is governed by pragmatic constraints such as point-of-view rather than purely structural constraints. In fact, the LTAG analysis proposed here is a relatively clean structural account of English binding data. The (un)availability of a derivation for certain examples may thus point to their classification into "exempt" and regular anaphora. These considerations are left for further work.

\subsection{Extraction}

A potential problem for the proposed analysis is presented by extraction phenomena, as in whmovement or topicalization. Extraction of a phrase containing an anaphor, whether topicalization or (17) or wh-movement (18), does not induce a Condition A violation. The current proposal predicts the grammaticality of (17a) and (18a) given that in each case the reflexive is locally ccommanded by its antecedent. However, in (17b) and (18b) the reflexive fails to be c-commanded by its antecedent, hence these examples are predicted to be ungrammatical although they are clearly acceptable.

a. $\operatorname{John}_{i}$ saw himself $_{i}$.

b. Himself ${ }_{i}$ John saw $\mathrm{t}_{i}$.

a. $\mathrm{John}_{i}$ liked the color pictures of himself $_{i}$.

b. [Which pictures of himself $i$ ] did John ${ }_{i}$ like $\varepsilon$ ?

A classical solution to these facts involves reconstruction of the $\mathrm{A}^{\prime}$-moved element to its original site for the purposes of binding. Clearly, syntactic reconstruction is untenable in LTAG. However, it is possible to emulate it through an entry akin to that in Figure 8, which is capable of deriving the topicalization examples in (17). The first component is the extracted reflexive 


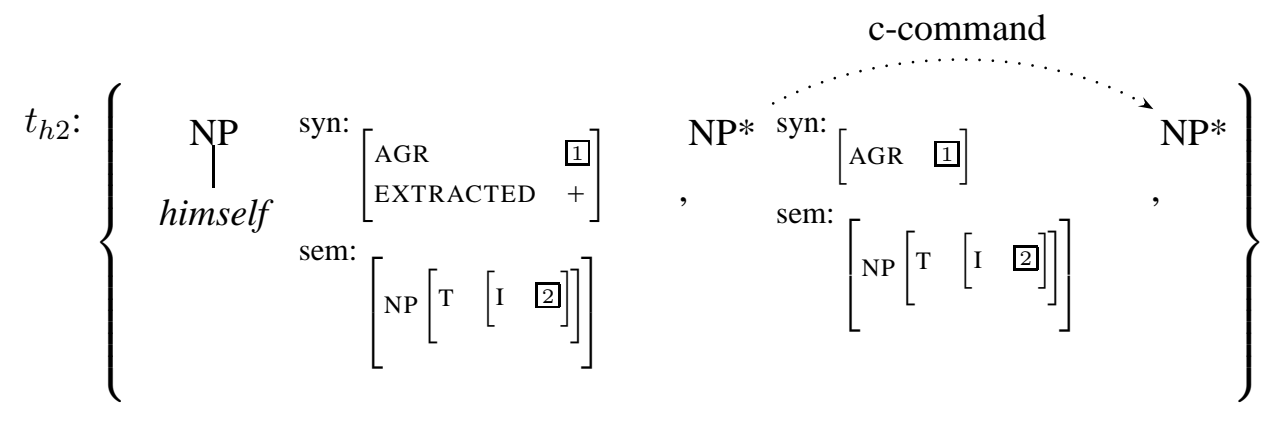

Figure 8: Lexical entry for extracted reflexive himself.

$\left(\mathrm{A}^{\prime}\right.$-moved constituents are marked by extractionfeatures (XTAG Group, 2001)), the second component is the binder, and the third component is the position that the reflexive has been extracted from. The requirement that the antecedent locally c-command the trace of movement has the effect of emulating reconstruction.

Note, furthermore, that even if some manner of reconstruction operation were to be implemented in LTAG, we are faced with the reality of cases such as (19), which demonstrate that extraction of an element can alter its set of possible binders. GB accounts (van Riemsdijk and Williams, 1986; Clark, 1982) have explained the opposition in (19) by allowing partial reconstruction to an intermediate trace from which the matrix subject is an accessible binder of the anaphor. The LTAG analysis of wh-movement, though, neither exploits intermediate traces nor allows transformational movement over domains larger than an elementary tree, meaning that such intermediate traces are simply unavailable to us.

$$
\begin{aligned}
& \text { a. }{ }^{*} \text { Marsha }_{i} \text { thought that I painted a } \\
& \text { picture of herself } f_{i} \text {. } \\
& \text { b. [Which pictures of herself }{ }_{i} \text { ] did } \\
& \text { Marsha }{ }_{i} \text { think that I painted } \varepsilon \text { ? }
\end{aligned}
$$

Instead, we suggest that Spec,IP subjects of clauses are able to bind into Spec, $\mathrm{CP}$ of the same clause as proposed by Reinhart (1991) and Frank and Kroch (1995). Rather than being a disadvantage, though, this seems to be a strength, predicting as it does that (20) is bad where reconstruction to a posited intermediate trace would predict acceptability.

(20) $*[$ Which pictures of himself $i$ ] did Mary think that $\mathrm{John}_{i}$ believed that Sally wanted?

Future work should attempt to determine the correct form of this lexical entry as well as whether or not it is possible to collapse it with the previously proposed Figure 8.

\subsection{Conditions B,C}

It is often assumed that the analyses for anaphors and regular pronouns should be related, because of a certain complementarity in distribution: While anaphors must be locally bound, pronouns must be locally free. In English, however, this complementarity is not absolute (cf. 21-22). Furthermore, a negative locality constraint seems to be discouraged by the LTAG framework. This suggests that the analysis of pronouns is independent of our account of anaphors. We leave pronouns, as well as r-expressions (Mary, the man) for further work.

(21) $\operatorname{John}_{i}$ pulled the blanket over him ${ }_{i} /$ himself ${ }_{i}$.

(22) a. They ${ }_{i}$ saw each other ${ }_{i}$ 's friends.

b. They ${ }_{i}$ saw their ${ }_{i}$ friends.

\subsection{Importance of the Left Edge}

Examination of language exposes the left edge to be special with regards to certain phenomena. In Binding Theory, this is revealed in the guise of a Subject Intervention Constraint. Case assignment represents a similar case. We see that verbs can assign accusative case to objects, and subjects of the next lowest clause (ECM), but no further. Ideally, a new analysis of left-edge effects would clarify the relationship between the two components of the lexical entry proposed above.

\subsection{Inherent Reflexives}

English has a small number of inherently reflexive verbs, such as behave:

(23) John behaves himself. ${ }^{5}$

Note that this verb requires its object to be a reflexive pronoun which is coreferent with its subject:

\footnotetext{
${ }^{5} \mathrm{We}$ would like to thank one anonymous reviewer for bringing this example to our attention.
} 
(24) * John behaves her.

We conclude from this that behave has a specific lexical constraint, namely that its object should be [+ reflexive]. Since there can be no other binder for this reflexive pronoun, it must be bound by the subject of the sentence.

\section{Conclusion}

In conclusion, we have presented an account of the syntax and semantics of anaphoric expressions that covers basic binding as well as raising, ECM, and extraction data. Our analysis employs a multicomponent lexical entry whose first component corresponds to the anaphor's binder, thus establishing a local relationship between antecedent and anaphor. A structural constraint that links the two components accounts for the basic asymmetry that is observed in the binding of reflexives and reciprocals in English.

\section{Acknowledgements}

We would like to thank the members of the XTAG group, as well as the CIS 630 class of Spring 2006 at Penn for comments and discussion.

\section{References}

Büring, Daniel. 2005. Binding Theory. Cambridge: Cambridge University Press.

Chomsky, Noam. 1981. Lectures on Government and Binding. Dordrecht: Foris.

Clark, Robin. 1982. Scope assignment and modification. Linguistic Inquiry 23: 1-28.

Copestake, Ann, Dan Flickinger, Ivan A. Sag, and Carl Pollard. 1999. Minimal Recursion Semantics: An introduction. Manuscript, Stanford University

Dalrymple, M., M. Kanazawa, Y. Kim, S.A. Mehombo, and S. Peters. 1998. Reciprocal expressions and the concept of reciprocity. Linguistics and Philosophy 21:159-210

Dimitriadis, Alexis. 2000. Syntactic locality and tree adjoining grammar: Grammatical, acquisition, and processing perspectives. Doctoral Dissertation, University of Pennsylvania.

Frank, Robert. 1992. Beyond Identity: Topics in Pronominal and Reciprocal Anaphora. Doctoral Dissertation, University of Pennsylvania.

Frank, Robert and Anthony Kroch. 1995. Generalized transformations and the theory of grammar. Studia Linguistica 49(2): 103-151.
Jackendoff, Ray. 1972. Semantic Interpretation in Generative Grammar. Cambridge, MA: MIT Press

Joshi, Aravind K., Laura Kallmeyer, and Maribel Romero. 2003. Flexible Composition in LTAG: Quantifier Scope and Inverse Linking. In Proceedings of the International Workshop on Compositional Semantics. Tilburg, The Netherlands

Joshi, Aravind K. and K. Vijay-Shanker. 1999. Compositional Semantics with Lexicalized TreeAdjoining Grammar (LTAG): How Much Underspecification is Necessary? In H.C. Blunt and E.G.C. Thijsse, editors. Proceedings of the Third International Workshop on Computational Semantics (IWCS-3), pp. 131-145. Tilburg, The Netherlands

Kallmeyer, Laura and Aravind K. Joshi. 2003. Factoring predicate argument and scope semantics: Underspecified semantics with LTAG. Research on Language and Computation 1:3-58

Kallmeyer, Laura and Maribel Romero. 2004. LTAG semantics with semantic unification. In Proceedings of $T A G+7$. Vancouver, Canada

Kuroda, S.Y.. 1965. Generative Gramamtical Studies in the Japanese Language. MIT: PhD. dissertation.

Pollard, Carl and Ivan Sag. 1994. Head-driven Phrase Structure Grammar. Chicago, Il: University of Chicago Press.

Postal, Paul. 1971. Crossover Phenomena. New York: Holt.

Reinhart, Tanya. 1991. Definite NP anaphora and ccommand domains. Linguistic Inquiry 12(4): 605635 .

Reuland, Eric and Martin Everaert. 2001. Deconstructing Binding. In M. Baltin and C. Collins, editors. The Handbook of Contemporary Syntactic Theory. Oxford

van Riemsdijk, Henk and Edwin Williams. 1986. Introduction to the Theory of Grammar. Cambridge, MA: MIT Press

XTAG Group. 2001. A Lexicalized Tree Adjoining Grammar for English. IRCS Technical Report, University of Pennsylvania 